\title{
Classes of Harmonic Functions Defined by Subordination
}

\author{
Jacek Dziok \\ Faculty of Mathematics and Natural Sciences, University of Rzeszów, Ulica Prof. Stanisława Pigonia 1, 35-310 Rzeszów, Poland
}

Correspondence should be addressed to Jacek Dziok; jdziok@ur.edu.pl

Received 13 July 2015; Accepted 5 October 2015

Academic Editor: Chengjian Zhang

Copyright (C) 2015 Jacek Dziok. This is an open access article distributed under the Creative Commons Attribution License, which permits unrestricted use, distribution, and reproduction in any medium, provided the original work is properly cited.

New classes of univalent harmonic functions are introduced. We give sufficient coefficient conditions for these classes. These coefficient conditions are shown to be also necessary if certain restrictions are imposed on the coefficients of these harmonic functions. By using extreme points theory we also obtain coefficients estimates, distortion theorems, and integral mean inequalities for these classes of functions. Radii of convexity and starlikeness of the classes are also considered.

\section{Introduction}

A continuous complex-valued function $f=u+i v$ defined in a simply connected domain $D \subset \mathbb{C}$ is said to be harmonic in $D$ if $u$ and $v$ are real harmonic in $D$. There is a close interrelation between analytic functions and harmonic functions. For real harmonic functions $u$ and $v$ there exist analytic functions $H$ and $G$ so that $u=\operatorname{Re}(H)$ and $v=\operatorname{Im}(G)$. Therefore, every complex-valued function $f$ harmonic in $D$ with $0 \in D$ can be uniquely represented as

$$
f=h+\bar{g},
$$

where $h=(H+G) / 2$ and $g=(H-G) / 2$ are analytic functions in $D$ with $g(0)=0$. Then we call $h$ the analytic part and $g$ the coanalytic part of $f$. It is easy to verify that the Jacobian of $f$ is given by

$$
J_{f}(z)=\left|h^{\prime}(z)\right|^{2}-\left|g^{\prime}(z)\right|^{2}, \quad(z \in D) .
$$

The mapping $f$ is locally univalent if $J_{f}(z) \neq 0$ in $D$. A result of Lewy [1] shows that the converse is true for harmonic mappings. Therefore, $f$ is locally univalent and sense-preserving if and only if

$$
\left|h^{\prime}(z)\right|>\left|g^{\prime}(z)\right|, \quad(z \in D) .
$$

Let $\mathscr{H}$ denote the class of harmonic functions in the unit $\operatorname{disc} \mathbb{U}:=\mathbb{U}(1)$, where $\mathbb{U}(r):=\{z \in \mathbb{C}:|z|<r\}$, and by $\mathscr{H}_{0}$ we denote the class of function $f \in \mathscr{H}$ normalized by $f(0)=$ $f_{z}^{\prime}(0)-1=0$. Then we may express the analytic functions $h$ and $g$ defined by (1) as

$$
\begin{aligned}
& h(z)=z+\sum_{n=2}^{\infty} a_{n} z^{n}, \\
& g(z)=\sum_{n=1}^{\infty} b_{n} z^{n},
\end{aligned}
$$

$$
\left(z \in \mathbb{U},\left|b_{1}\right|<1\right) \text {; }
$$

that is,

$$
\begin{aligned}
f(z)=\sum_{n=1}^{\infty}\left(a_{n} z^{n}+\overline{b_{n} z^{n}}\right), & \\
& \left(a_{1}=1, \quad\left|b_{1}\right|<1, z \in \mathbb{U}\right) .
\end{aligned}
$$

By $\mathcal{S}_{\mathscr{H}}$ we denote the class of functions $f \in \mathscr{H}_{0}$ which are univalent and sense-preserving in $\mathbb{U}$, and by $\mathscr{A}$ we denote the class of functions $f \in \mathscr{H}$ for which the coanalytic part vanishes.

We say that a function $f: \mathbb{U} \rightarrow \mathbb{C}$ is subordinate to a function $F: \mathbb{U} \rightarrow \mathbb{C}$ and write $f(z) \prec F(z)$ (or simply $f \prec$ $F$ ), if there exists a complex-valued function $\omega$ which maps $\mathbb{U}$ into oneself with $\omega(0)=0$, such that

$$
f(z)=F(\omega(z)), \quad(z \in \mathbb{U}) .
$$


In particular, if $F$ is univalent in $\mathbb{U}$, we have the following equivalence:

$$
f(z) \prec F(z) \Longleftrightarrow[f(0)=F(0), f(\mathbb{U}) \subset F(\mathbb{U})] .
$$

For functions $f_{1}, f_{2} \in \mathscr{H}$ of the form

$$
f_{k}(z)=\sum_{n=0}^{\infty} a_{k, n} z^{n}+\sum_{n=1}^{\infty} \overline{b_{k, n}} \bar{z}^{n}
$$

$$
(z \in \mathbb{U}, k \in \mathbb{N}:=\{1,2\})
$$

by $f_{1} * f_{2}$ we denote the Hadamard product or convolution of $f_{1}$ and $f_{2}$, defined by

$$
\left(f_{1} * f_{2}\right)(z)=\sum_{n=0}^{\infty} a_{1, n} a_{2, n} z^{n}+\sum_{n=1}^{\infty} \overline{b_{1, n} b_{2, n}} \bar{z}^{n}, \quad(z \in \mathbb{U}) .
$$

Firstly, Clunie and Sheil-Small [2] and Sheil-Small [3] studied $\mathcal{S}_{\mathscr{H}}$ together with some of its geometric subclasses. In particular, they investigated harmonic starlike functions in $\mathbb{U}$ and harmonic convex functions in $\mathbb{U}$, which are defined as follows. We say that $f \in \mathcal{S}_{\mathscr{H}}$ is said to be harmonic starlike functions in $\mathbb{U}(r)$ if $f(\mathbb{U}(r))$ is a starlike domain with respect to the origin. Likewise $f \in \mathcal{S}_{\mathscr{H}}$ is said to be harmonic convex functions in $\mathbb{U}(r)$ if $f(\mathbb{U}(r))$ is a convex domain.

In particular, we have that $f \in \mathscr{H}_{0}$ is harmonic starlike function if

$$
\frac{\partial}{\partial t}\left(\arg f\left(r e^{i t}\right)\right)>0, \quad 0 \leq t \leq 2 \pi, 0<r<1,
$$

or equivalently

$$
\operatorname{Re} \frac{D_{\mathscr{H}} f(z)}{f(z)}>0, \quad(z \in \mathbb{U})
$$

where

$$
D_{\mathscr{H}} f(z):=z h^{\prime}(z)-\overline{z g^{\prime}(z)}, \quad(z \in \mathbb{U}) .
$$

Let $-B \leq A<B \leq 1,0 \leq \alpha<1$. Motivated by Janowski [4] we define the following classes of functions. that

Let $\mathcal{S}_{\mathscr{H}}^{*}(A, B)$ denote the class of functions $f \in \mathcal{S}_{\mathscr{H}}$ such

$$
\frac{D_{\mathscr{\ell}} f(z)}{f(z)} \prec \frac{1+A z}{1+B z}
$$

Also, by $\mathscr{R}_{\mathscr{H}}(A, B)$ we denote the class of functions $f \in \mathcal{S}_{\mathscr{H}}$ such that

$$
\frac{D_{\mathscr{\ell}} f(z)}{z} \prec \frac{1+A z}{1+B z} .
$$

Moreover, let us define

$$
\begin{aligned}
\mathcal{S}_{\mathscr{H}}^{c}(A, B) & :=\left\{f \in \mathcal{S}_{\mathscr{H}}: D_{\mathscr{H}} f \in \mathcal{S}_{\mathscr{H}}^{*}(A, B)\right\}, \\
\mathscr{R}_{\mathscr{H}}^{1}(A, B) & :=\left\{f \in \mathcal{S}_{\mathscr{H}}: D_{\mathscr{H}} f \in \mathscr{R}_{\mathscr{H}}(A, B)\right\}, \\
\mathcal{S}_{\mathscr{H}}^{*}(\alpha) & :=\mathcal{S}_{\mathscr{H}}^{*}(2 \alpha-1,1), \\
\mathcal{S}_{\mathscr{H}}^{c}(\alpha) & :=\mathcal{S}_{\mathscr{H}}^{c}(2 \alpha-1,1) .
\end{aligned}
$$

We should notice here that Janowski [4] introduced the classes $\mathcal{S}^{*}(A, B):=\mathcal{S}_{\mathscr{H}}^{*}(A, B) \cap \mathscr{A}$ and $\mathscr{R}(A, B)$ := $\mathscr{R}_{\mathscr{H}}(A, B) \cap \mathscr{A}$. The classes $\mathcal{S}_{\mathscr{H}}^{*}(\alpha)$ and $\mathcal{S}_{\mathscr{H}}^{c}(\alpha)$ were investigated by Jahangiri $[5,6]$. The classes $\mathcal{S}_{\mathscr{H}}^{*}:=\mathcal{S}_{\mathscr{H}}^{*}(0)$ and $\mathcal{S}_{\mathscr{H}}^{c}:=\mathcal{S}_{\mathscr{H}}^{c}(0)$ are the classes of functions $f \in \mathcal{S}_{\mathscr{H}}$ which are starlike in $\mathbb{U}(r)$ or convex in $\mathbb{U}(r)$, respectively, for all $r \in(0,1\rangle$. It is easy to verify (e.g., see [5]) that

$$
\begin{aligned}
& \delta_{\mathscr{H}}^{c}(A, B) \subset \mathcal{S}_{\mathscr{H}}^{c} \subset \mathcal{S}_{\mathscr{H}}^{*} \subset \mathcal{S}_{\mathscr{H}}, \\
& \mathcal{S}_{\mathscr{H}}^{*}(A, B) \subset \mathcal{S}_{\mathscr{H}}^{*} .
\end{aligned}
$$

In the paper we obtain some necessary and sufficient conditions for defined classes of functions. Some topological properties, radii of convexity and starlikeness, and extreme points of the classes are also considered. By using extreme points theory we obtain coefficients estimates, distortion theorems, and integral mean inequalities for the classes of functions.

\section{Necessary and Sufficient Conditions}

Theorem 1. Let $f \in \mathcal{S}_{\mathscr{H}}$. Then $f \in \mathcal{S}_{\mathscr{H}}^{*}(A, B)$ if and only if

$$
f(z) * \varphi(z ; \xi) \neq 0, \quad(\xi \in \mathbb{C},|\xi|=1),
$$

where

$$
\begin{aligned}
\varphi(z ; \xi)= & \frac{(B-A) \xi z+(1+A \xi) z^{2}}{(1-z)^{2}} \\
& -\frac{\{2+(A+B) \xi\} \bar{z}-(1+A \xi) \bar{z}^{2}}{(1-\bar{z})^{2}},
\end{aligned}
$$

$(z \in \mathbb{U})$

Proof. Let $f \in \mathcal{S}_{\mathscr{H}}$ be of form (1). Then $f \in \mathcal{S}_{\mathscr{H}}^{*}(A, B)$ if and only if it satisfies (13) or equivalently

$$
\frac{D_{\mathscr{H}} f(z)}{f(z)} \neq \frac{1+A \xi}{1+B \xi}, \quad(\xi \in \mathbb{C}, \quad|\xi|=1) .
$$

Since

$$
\begin{aligned}
z h^{\prime}(z) & =h(z) * \frac{z}{(1-z)^{2}}, \\
h(z) & =h(z) * \frac{z}{1-z},
\end{aligned}
$$

we have

$$
\begin{aligned}
(1+B \xi) D_{\mathscr{H}} f(z)-(1+A \xi) f(z) & \\
= & (1+B \xi) z h^{\prime}(z)-(1+A \xi) h(z) \\
& -\left[(1+B \xi) \overline{z g^{\prime}(z)}+(1+A \xi) \overline{g(z)}\right] \\
= & h(z) *\left(\frac{(1+B \xi) z}{(1-z)^{2}}-\frac{(1+A \xi) z}{1-z}\right)-\overline{g(z)} \\
& *\left(\frac{(1+B \xi) \bar{z}}{(1-\bar{z})^{2}}+\frac{(1+A \xi) \bar{z}}{1-\bar{z}}\right) \\
= & f(z) * \varphi(z ; \xi) .
\end{aligned}
$$


Thus, conditions (17) and (19) are equivalent, and the proof is completed.

Putting $B=-A=1$ in Theorem 1 we obtain the following corollary.

Corollary 2. Let $f \in \mathcal{S}_{\mathscr{H}}$. Then $f \in \mathcal{S}_{\mathscr{H}}^{*}$ if and only if

$$
f(z) * \varphi(z ; \xi) \neq 0, \quad(\xi \in \mathbb{C},|\xi|=1),
$$

where

$$
\varphi(z ; \xi)=\frac{2 \xi z+(1-\xi) z^{2}}{(1-z)^{2}}-\frac{2 \bar{z}-(1-\xi) \bar{z}^{2}}{(1-\bar{z})^{2}},
$$

$(z \in \mathbb{U})$.

Theorem 3. If a function $f \in \mathscr{H}_{0}$ of form (5) satisfies the condition

$$
\sum_{n=1}^{\infty}\left(\alpha_{n}\left|a_{n}\right|+\beta_{n}\left|b_{n}\right|\right) \leq 2(B-A),
$$

where

$$
\begin{aligned}
& \alpha_{n}=n(1+B)-(1+A), \\
& \beta_{n}=n(1+B)+(1+A),
\end{aligned}
$$

then $f \in \mathcal{S}_{\mathscr{H}}^{*}(A, B)$.

Proof. It is clear that the theorem is true for the function $f(z) \equiv z$. Let $f \in \mathscr{H}_{0}$ be a function of form (5) and let there exist $n \in \mathbb{N}$ such that $a_{n} \neq 0(n \geq 2)$ or $b_{n} \neq 0$. Since

$$
\begin{aligned}
& \frac{\alpha_{n}}{B-A} \geq n, \\
& \frac{\beta_{n}}{B-A} \geq n, \\
& \quad n \in \mathbb{N},
\end{aligned}
$$

by (24) we have

$$
\begin{aligned}
& \sum_{n=2}^{\infty} n\left(\left|a_{n}\right|+\left|b_{n}\right|\right) \leq 1-b_{1}, \\
& \left|h^{\prime}(z)\right|-\left|g^{\prime}(z)\right| \geq 1-b_{1}-\sum_{n=2}^{\infty} n\left(\left|a_{n}\right|+\left|b_{n}\right|\right)|z|^{n-1} \\
& \quad \geq 1-b_{1}-|z| \sum_{n=2}^{\infty}\left(n\left|a_{n}\right|+n\left|b_{n}\right|\right)\left(1-b_{1}\right)(1-|z|) \\
& \quad>0, \quad(z \in \mathbb{U}) .
\end{aligned}
$$

Thus, by (3) function $f$ is locally univalent and sense-preserving in $\mathbb{U}$. Moreover, if $z_{1}, z_{2} \in \mathbb{U}, z_{1} \neq z_{2}$, then

$$
\begin{array}{r}
\left|\frac{z_{1}^{n}-z_{2}^{n}}{z_{1}-z_{2}}\right|=\left|\sum_{k=1}^{n} z_{1}^{k-1} z_{2}^{n-k}\right| \leq \sum_{k=1}^{n}\left|z_{1}\right|^{k-1}\left|z_{2}\right|^{n-k}<n, \\
(n=2,3, \ldots) .
\end{array}
$$

Therefore, by (27) we have

$$
\begin{aligned}
& \left|f\left(z_{1}\right)-f\left(z_{2}\right)\right| \geq\left|h\left(z_{1}\right)-h\left(z_{2}\right)\right| \\
& -\left|g\left(z_{1}\right)-g\left(z_{2}\right)\right| \geq\left|z_{1}-z_{2}+\sum_{n=2}^{\infty} a_{n}\left(z_{1}^{n}-z_{2}^{n}\right)\right| \\
& -\left|\sum_{n=1}^{\infty} \overline{b_{n}\left(z_{1}^{n}-z_{2}^{n}\right)}\right| \geq\left|z_{1}-z_{2}\right|-\sum_{n=2}^{\infty}\left|a_{n}\right|\left|z_{1}^{n}-z_{2}^{n}\right| \\
& -\sum_{n=1}^{\infty}\left|b_{n}\right|\left|z_{1}^{n}-z_{2}^{n}\right|=\left|z_{1}-z_{2}\right| \\
& \cdot\left(1-b_{1}-\sum_{n=2}^{\infty}\left|a_{n}\right|\left|\frac{z_{1}^{n}-z_{2}^{n}}{z_{1}-z_{2}}\right|-\sum_{n=2}^{\infty}\left|b_{n}\right|\left|\frac{z_{1}^{n}-z_{2}^{n}}{z_{1}-z_{2}}\right|\right) \\
& >\left|z_{1}-z_{2}\right|\left(1-b_{1}-\sum_{n=2}^{\infty} n\left(\left|a_{n}\right|+\left|b_{n}\right|\right)\right) \geq 0 .
\end{aligned}
$$

This leads to the univalence of $f$; that is, $f \in \mathcal{S}_{\mathscr{H}}$. Therefore, $f \in \mathcal{S}_{\mathscr{H}}^{*}(A, B)$ if and only if there exists a complex-valued function $\omega, \omega(0)=0,|\omega(z)|<1(z \in \mathbb{U})$, such that

$$
\frac{D_{\mathscr{L}} f(z)}{f(z)}=\frac{1+A \omega(z)}{1+B \omega(z)} \quad(z \in \mathbb{U}),
$$

or equivalently

$$
\left|\frac{D_{\mathscr{H}} f(z)-f(z)}{B D_{\mathscr{H}} f(z)-A f(z)}\right|<1, \quad(z \in \mathbb{U}) .
$$

Thus, it is sufficient to prove that

$$
\begin{aligned}
\left|D_{\mathscr{H}} f(z)-f(z)\right|-\left|B D_{\mathscr{H}} f(z)-A f(z)\right| & <0, \\
& (z \in \mathbb{U} \backslash\{0\}) .
\end{aligned}
$$

Indeed, letting $|z|=r(0<r<1)$ we have

$$
\begin{aligned}
& \left|D_{\mathscr{H}} f(z)-f(z)\right|-\left|B D_{\mathscr{H}} f(z)-A f(z)\right| \\
& \quad=\left|\sum_{n=2}^{\infty}(n-1) a_{n} z^{n}-\sum_{n=1}^{\infty}(n+1) \bar{b}_{n} \bar{z}^{n}\right|-\mid(B-A) z \\
& \quad+\sum_{n=2}^{\infty}(B n-A) a_{n} z^{n}-\sum_{n=1}^{\infty}(B n+A) \bar{b}_{n} \bar{z}^{n} \mid \\
& \quad \leq \sum_{n=2}^{\infty}(n-1)\left|a_{n}\right| r^{n}+\sum_{n=1}^{\infty}(n+1)\left|b_{n}\right| r^{n}-(B-A) r \\
& \quad+\sum_{n=2}^{\infty}(B n-A)\left|a_{n}\right| r^{n}+\sum_{n=1}^{\infty}(B n+A)\left|b_{n}\right| r^{n} \\
& \quad \leq r\left\{\sum_{n=1}^{\infty}\left(\alpha_{n}\left|a_{n}\right|+\beta_{n}\left|b_{n}\right|\right) r^{n-1}-2(B-A)\right\}<0,
\end{aligned}
$$

whence $f \in \mathcal{S}_{\mathscr{H}}^{*}(A, B)$. 
Motivated by Silverman [7] we denote by $\mathscr{T}^{\lambda}(\lambda \in\{0,1\})$ the class of functions $f \in \mathscr{H}_{0}$ of form (5) such that $a_{n}=-\left|a_{n}\right|$, $b_{n}=(-1)^{\lambda}\left|b_{n}\right|(n=2,3, \ldots)$; that is,

$$
\begin{gathered}
f=h+\bar{g}, \\
h(z)=z-\sum_{n=2}^{\infty}\left|a_{n}\right| z^{n}, \\
g(z)=(-1)^{\lambda} \sum_{n=1}^{\infty}\left|b_{n}\right| \bar{z}^{n},
\end{gathered}
$$

$(z \in \mathbb{U})$

Moreover, let us define

$$
\begin{aligned}
\mathcal{S}_{\mathscr{T}}^{*}(A, B) & :=\mathscr{T}^{0} \cap \mathcal{S}_{\mathscr{H}}^{*}(A, B), \\
\mathcal{S}_{\mathscr{T}}^{c}(A, B) & :=\mathscr{T}^{1} \cap \mathcal{S}_{\mathscr{H}}^{c}(A, B), \\
\mathscr{R}_{\mathscr{T}}(A, B) & :=\mathscr{T}^{0} \cap \mathscr{R}_{\mathscr{H}}(A, B), \\
\mathscr{R}_{\mathscr{T}}^{1}(A, B) & :=\mathscr{T}^{1} \cap \mathscr{R}_{\mathscr{H}}^{1}(A, B), \\
\mathcal{S}_{\mathscr{T}}^{*} & :=\mathcal{S}_{\mathscr{T}}^{*}(-1,1), \\
\mathcal{S}_{\mathscr{T}}^{c} & :=\mathcal{S}_{\mathscr{T}}^{c}(-1,1) .
\end{aligned}
$$

Now, we show that condition (24) is also the sufficient condition for a function $f \in \mathscr{T}^{0}$ to be in class $\mathcal{S}_{\mathscr{T}}^{*}(A, B)$.

Theorem 4. Let $f \in \mathscr{T}^{0}$ be a function of form (35). Then $f \in$ $\mathcal{S}_{\mathscr{T}}^{*}(A, B)$ if and only if condition (24) holds true.

Proof. In view of Theorem 3 we need only to show that each function $f \in \mathcal{S}_{\mathscr{T}}^{*}(A, B)$ satisfies the coefficient inequality (24). If $f \in \mathcal{S}_{\mathscr{T}}^{*}(A, B)$, then it satisfies (32) or equivalently

$$
\begin{aligned}
& \left|\frac{\sum_{n=1}^{\infty}\left\{(n-1)\left|a_{n}\right| z^{n}+(n+1)\left|b_{n}\right| \bar{z}^{n}\right\}}{2(B-A) z-\sum_{n=1}^{\infty}\left\{(B n-A)\left|a_{n}\right| z^{n}+(B n+A)\left|b_{n}\right| \bar{z}^{n}\right\}}\right| \\
& \quad<1, \quad(z \in \mathbb{U}) .
\end{aligned}
$$

Therefore, putting $z=r(0 \leq r<1)$ we obtain

$$
\begin{aligned}
& \frac{\sum_{n=1}^{\infty}\left\{(n-1)\left|a_{n}\right|+(n+1)\left|b_{n}\right| r^{n-1}\right\}}{2(B-A)-\sum_{n=1}^{\infty}\left\{(B n-A)\left|a_{n}\right|+(B n+A)\left|b_{n}\right| r^{n-1}\right\}} \\
& <1 .
\end{aligned}
$$

It is clear that the denominator of the left hand side cannot vanish for $r \in(0,1)$. Moreover, it is positive for $r=0$, and in consequence for $r \in(0,1)$. Thus, by (38) we have

$$
\sum_{n=1}^{\infty}\left(\alpha_{n}\left|a_{n}\right|+\beta_{n}\left|b_{n}\right|\right) r^{n-1}<B-A, \quad(0<r<1) .
$$

The sequence of partial sums $\left\{S_{n}\right\}$ associated with the series $\sum_{n=2}^{\infty}\left(\alpha_{n}\left|a_{n}\right|+\beta_{n}\left|b_{n}\right|\right)$ is nondecreasing sequence. Moreover, by (39) it is bounded by $B-A$. Hence, the sequence $\left\{S_{n}\right\}$ is convergent and

$$
\sum_{n=2}^{\infty}\left(\alpha_{n}\left|a_{n}\right|+\beta_{n}\left|b_{n}\right|\right)=\lim _{n \rightarrow \infty} S_{n} \leq B-A,
$$

which yields assertion (24).

By using Theorems 1-4 and definition of the class $\mathcal{S}_{\mathscr{H}}^{c}(A, B)$ we have the following three corollaries.

Corollary 5. Let $f \in \mathcal{S}_{\mathscr{H}}$. Then $f \in \mathcal{S}_{\mathscr{H}}^{c}(A, B)$ if and only if

$$
D_{\mathscr{H}} f(z) * \varphi(z ; \xi) \neq 0, \quad(|\xi|=1),
$$

where $\varphi(z ; \xi)$ is defined by (18).

Corollary 6. If a function $f \in \mathscr{H}_{0}$ of form (5) satisfies the condition

$$
\sum_{n=1}^{\infty}\left(n \alpha_{n}\left|a_{n}\right|+n \beta_{n}\left|b_{n}\right|\right) \leq 2(B-A),
$$

then $f \in \mathcal{S}_{\mathscr{H}}^{c}(A, B)$.

Corollary 7. Let $f \in \mathscr{T}^{1}$ be a function of form (35). Then $f \in \mathcal{S}_{\mathscr{T}}^{c}(A, B)$ if and only if condition (42) holds true.

Similar to Theorem 4 and Corollary 6 we can obtain the following two results.

Theorem 8. Let $f \in \mathscr{T}^{0}$ be a function of form (35). Then $f \in$ $\mathscr{R}_{\mathscr{T}}(A, B)$ if and only if

$$
\sum_{n=1}^{\infty} n\left(\left|a_{n}\right|+\left|b_{n}\right|\right) \leq \frac{2 B-A+1}{1+B} .
$$

Corollary 9. Let $f \in \mathscr{T}^{1}$ be a function of form (35). Then $f \in \mathscr{R}_{\mathscr{T}}^{1}(A, B)$ if and only if

$$
\sum_{n=1}^{\infty} n^{2}\left(\left|a_{n}\right|+\left|b_{n}\right|\right) \leq \frac{2 B-A+1}{1+B} .
$$

Thus we have the following.

Corollary 10. Consider

$$
\begin{aligned}
& \mathcal{S}_{\mathscr{T}}^{*}=\mathscr{R}_{\mathscr{T}}:=\mathscr{R}_{\mathscr{T}}(-1,1), \\
& \mathcal{S}_{\mathscr{T}}^{c}=\mathscr{R}_{\mathscr{T}}^{1}:=\mathscr{R}_{\mathscr{T}}^{1}(-1,1) .
\end{aligned}
$$

\section{Radii of Starlikeness and Convexity}

We say that a function $f \in \mathscr{H}_{0}$ is starlike of order $\alpha$ in $\mathbb{U}(r)$ if

$$
\frac{\partial}{\partial t}\left(\arg f\left(\rho e^{i t}\right)\right)>\alpha, \quad 0 \leq t \leq 2 \pi, 0<\rho<r<1 .
$$

Analogously, we say that a function $f \in \mathscr{H}_{0}$ is convex of order $\alpha$ in $\mathbb{U}(r)$ if

$$
\frac{\partial}{\partial t}\left(\arg \frac{\partial}{\partial t} f\left(\rho e^{i t}\right)\right)>\alpha
$$


It is easy to verify that for a function $f \in \mathscr{T}$ condition (46) is equivalent to

$$
\operatorname{Re} \frac{D_{\mathscr{H}} f(z)}{f(z)}>\alpha, \quad(z \in \mathbb{U}(r))
$$

or equivalently

$$
\left|\frac{D_{\mathscr{H}} f(z)-(1+\alpha) f(z)}{D_{\mathscr{H}} f(z)+(1-\alpha) f(z)}\right|<1, \quad(z \in \mathbb{U}(r)) .
$$

Let $\mathscr{B}$ be a subclass of the class $\mathscr{H}_{0}$. We define the radius of starlikeness $R_{\alpha}^{*}(\mathscr{B})$ and the radius of convexity $R_{\alpha}^{c}(\mathscr{B})$ for the class $\mathscr{B}$ by

$$
\begin{aligned}
& R_{\alpha}^{*}(\mathscr{B}) \\
& :=\inf _{f \in \mathscr{B}}(\sup \{r \in(0,1]: f \text { is starlike of order } \alpha \text { in } \mathbb{U}(r)\}), \\
& R_{\alpha}^{c}(\mathscr{B}) \\
& :=\inf _{f \in \mathscr{B}}(\sup \{r \in(0,1]: f \text { is convex of order } \alpha \text { in } \mathbb{U}(r)\}) .
\end{aligned}
$$

Theorem 11. The radius of starlikeness of order $\alpha$ for the class $\mathcal{S}_{\mathscr{T}}^{*}(A, B), 0 \leq \alpha \leq(2+2 A) /(2+A+3 B)$, is given by

$$
\begin{aligned}
R_{\alpha}^{*} & \left(\mathcal{S}_{\mathscr{T}}^{*}(A, B)\right) \\
& =\inf _{n \geq 2}\left(\frac{1-\alpha}{B-A} \min \left\{\frac{\alpha_{n}}{n-\alpha}, \frac{\beta_{n}}{n+\alpha}\right\}\right)^{1 /(n-1)},
\end{aligned}
$$

where $\alpha_{n}$ and $\beta_{n}$ are defined by (25).

Proof. Let $f \in \mathcal{S}_{\mathscr{T}}^{*}(A, B)$ be of form (35). Then, for $|z|=r<1$ we have

$$
\begin{aligned}
& \left|\frac{D_{\mathscr{H}} f(z)-(1+\alpha) f(z)}{D_{\mathscr{H}} f(z)+(1-\alpha) f(z)}\right| \\
& =\left|\frac{\alpha z-\sum_{n=2}^{\infty}(n-1-\alpha)\left|a_{n}\right| z^{n}+\sum_{n=1}^{\infty}(n+1+\alpha)\left|b_{n}\right| \bar{z}^{n}}{(2-\alpha) z-\sum_{n=2}^{\infty}(n+1-\alpha)\left|a_{n}\right| z^{n}+\sum_{n=1}^{\infty}(n-1+\alpha)\left|b_{n}\right| \bar{z}^{n}}\right| \\
& \leq \frac{2 \alpha+\sum_{n=1}^{\infty}\left((n-1-\alpha)\left|a_{n}\right|+(n+1+\alpha)\left|b_{n}\right|\right) r^{n-1}}{4-2 \alpha-\sum_{n=1}^{\infty}\left((n+1-\alpha)\left|a_{n}\right|+(n-1+\alpha)\left|b_{n}\right|\right) r^{n-1}} .
\end{aligned}
$$

Thus, condition (49) is true if and only if

$$
\sum_{n=1}^{\infty}\left(\frac{n-\alpha}{1-\alpha}\left|a_{n}\right|+\frac{n+\alpha}{1-\alpha}\left|b_{n}\right|\right) r^{n-1} \leq 2 .
$$

By Theorem 4, we have

$$
\sum_{n=1}^{\infty}\left(\frac{\alpha_{n}}{B-A}\left|a_{n}\right|+\frac{\beta_{n}}{B-A}\left|b_{n}\right|\right) \leq 2,
$$

where $\alpha_{n}$ and $\beta_{n}$ are defined by (25). Since $\alpha_{n}<\beta_{n}(n \in \mathbb{N})$, condition (53) is true if

$$
\begin{aligned}
& \frac{n-\alpha}{1-\alpha} r^{n-1} \leq \frac{\alpha_{n}}{B-A}, \\
& \frac{n+\alpha}{1-\alpha} r^{n-1} \leq \frac{\beta_{n}}{B-A},
\end{aligned}
$$

that is, if $(1+\alpha) /(1-\alpha) \leq \beta_{1} /(B-A)($ or $\alpha \leq(2+2 A) /(2+$ $A+3 B)$ ) and

$$
r \leq\left(\frac{1-\alpha}{B-A} \min \left\{\frac{\alpha_{n}}{n-\alpha}, \frac{\beta_{n}}{n+\alpha}\right\}\right)^{1 /(n-1)},
$$

$$
(n=2,3, \ldots) \text {. }
$$

It follows that function $f$ is starlike of order $\alpha$ in the disk $U\left(r^{*}\right)$, where $r^{*}$ is

$$
r^{*}:=\inf _{n \geq 2}\left(\frac{1-\alpha}{B-A} \min \left\{\frac{\alpha_{n}}{n-\alpha}, \frac{\beta_{n}}{n+\alpha}\right\}\right)^{1 /(n-1)}
$$

The radii of starlikeness $r^{*}\left(h_{n}^{*}\right)$ and $r^{*}\left(h_{n}^{*}\right)$ of functions $h_{n}^{*}, g_{n}^{*}(n=2,3, \ldots)$ of form (75) are given by

$$
\begin{aligned}
& r^{*}\left(h_{n}^{*}\right)=\left(\frac{1-\alpha}{n-\alpha} \frac{\alpha_{n}}{B-A}\right)^{1 /(n-1)}, \\
& r^{*}\left(g_{n}^{*}\right)=\left(\frac{1-\alpha}{n+\alpha} \frac{\beta_{n}}{B-A}\right)^{1 /(n-1)} .
\end{aligned}
$$

Therefore, the radius $r^{*}$ given by (57) cannot be larger. Thus we have (51).

The following result may be proved in much the same way as Theorem 11.

Theorem 12. Let $\alpha_{n}$ and $\beta_{n}$ be defined by (25). Then

$$
\begin{aligned}
& R_{\alpha}^{c}\left(\mathcal{S}_{\mathscr{T}}^{*}(A, B)\right)=R_{\alpha}^{*}\left(\mathcal{S}_{\mathscr{T}}^{c}(A, B)\right) \\
& =\inf _{n \geq 2}\left(\frac{1-\alpha}{B-A} \min \left\{\frac{\alpha_{n}}{n(n-\alpha)}, \frac{\beta_{n}}{n(n+\alpha)}\right\}\right)^{1 /(n-1)}, \\
& R_{\alpha}^{c}\left(\mathcal{S}_{\mathscr{T}}^{c}(A, B)\right) \\
& =\inf _{n \geq 2}\left(\frac{1-\alpha}{B-A} \min \left\{\frac{\alpha_{n}}{n^{2}(n-\alpha)}, \frac{\beta_{n}}{n^{2}(n+\alpha)}\right\}\right)^{1 /(n-1)} .
\end{aligned}
$$

\section{Topological Properties}

We consider the usual topology on $\mathscr{H}$ defined by a metric in which a sequence $\left\{f_{n}\right\}$ in $\mathscr{H}$ converges to $f$ if and only if it converges to $f$ uniformly on each compact subset of $\mathbb{U}$. It follows from the theorems of Weierstrass and Montel that this topological space is complete.

Let $\mathscr{F}$ be a subclass of the class $\mathscr{H}$. A function $f \in \mathscr{F}$ is called an extreme point of $\mathscr{F}$ if the condition

$$
f=\gamma f_{1}+(1-\gamma) f_{2}, \quad\left(f_{1}, f_{2} \in \mathscr{F}, 0<\gamma<1\right)
$$

implies $f_{1}=f_{2}=f$. We will use the notation $E \mathscr{F}$ to denote the set of all extreme points of $\mathscr{F}$. It is clear that $E \mathscr{F} \subset \mathscr{F}$.

We say that $\mathscr{F}$ is locally uniformly bounded if for each $r, 0<r<1$, there is a real constant $M=M(r)$ so that

$$
|f(z)| \leq M, \quad(f \in \mathscr{F},|z| \leq r) .
$$

We say that a class $\mathscr{F}$ is convex if

$$
\gamma f+(1-\gamma) g \in \mathscr{F}, \quad(f, g \in \mathscr{F}, 0 \leq \gamma \leq 1) .
$$


Moreover, we define the closed convex hull of $\mathscr{F}$ as the intersection of all closed convex subsets of $\mathscr{H}$ that contain $\mathscr{F}$. We denote the closed convex hull of $\mathscr{F}$ by $\overline{\mathrm{co}} \mathscr{F}$.

A real-valued functional $\mathscr{J}: \mathscr{H} \rightarrow \mathbb{R}$ is called convex on a convex class $\mathscr{F} \subset \mathscr{H}$ if

$$
\begin{aligned}
\mathscr{J}(\gamma f+(1-\gamma) g) \leq \gamma \mathscr{J}(f)+ & (1-\gamma) \mathscr{J}(g), \\
& (f, g \in \mathscr{F}, 0 \leq \gamma \leq 1) .
\end{aligned}
$$

The Krein-Milman theorem (see [8]) is fundamental in the theory of extreme points. In particular, it implies the following lemma.

Lemma 13. If $\mathscr{F}$ is a nonempty compact subclass of the class $\mathscr{H}$, then $E \mathscr{F}$ is nonempty and $\overline{\mathrm{co}} E \mathscr{F}=\overline{\mathrm{co}} \mathscr{F}$.

Due to Hallenbeck and MacGregor [9, pp. 45] (see also [10]) we prove the following result.

Lemma 14. Let $\mathscr{F}$ be a nonempty compact convex subclass of the class $\mathscr{H}$ and let $\mathscr{J}: \mathscr{H} \rightarrow \mathbb{R}$ be a real-valued, continuous, and convex functional on $\mathscr{F}$. Then

$$
\max \{\mathscr{J}(f): f \in \mathscr{F}\}=\max \{\mathscr{J}(f): f \in E \mathscr{F}\} .
$$

Proof. Since the functional $\mathscr{J}$ is continuous on the compact set $\mathscr{F}$ there exists

$$
\max \{\mathscr{J}(f): f \in \mathscr{F}\}=: M .
$$

Therefore, the set $G:=\{f \in \mathscr{F}: \mathscr{J}(f)=M\}$ is nonempty compact subclass of $\mathscr{F}$. Hence, by Lemma 13 we find that $G$ has an extreme point $f_{0}$. Suppose that

$$
f_{0}=\gamma f_{1}+(1-\gamma) f_{2}
$$

where $f_{1}, f_{2} \in \mathscr{F}$ and $0<\gamma<1$. Then

$$
\begin{aligned}
M & =\mathscr{J}\left(f_{0}\right) \leq \gamma \mathscr{J}\left(f_{1}\right)+(1-\gamma) \mathscr{J}\left(f_{2}\right) \\
& \leq \gamma M+(1-\gamma) M=M
\end{aligned}
$$

and we must have $\mathscr{J}\left(f_{1}\right)=\mathscr{J}\left(f_{2}\right)=M$; that is, $f_{1}, f_{2} \in G$. Since $f_{0}$ is an extreme point of $G$ we have $f_{1}=f_{2}=f_{0}$ and, in consequence, $f_{0} \in E \mathscr{F}$. Thus, we conclude that there exists $\max \{\mathscr{J}(f): f \in E \mathscr{F}\}=M$, and the proof is complete.

Since $\mathscr{H}$ is a complete metric space, Montel's theorem (see [11]) implies the following lemma.

Lemma 15. A class $\mathscr{F} \subset \mathscr{H}$ is compact if and only if $\mathscr{F}$ is closed and locally uniformly bounded.

Theorem 16. The class $\mathcal{S}_{\mathscr{T}}^{*}(A, B)$ is convex and compact subset of $\mathscr{H}_{0}$.

Proof. Let $f_{k} \in \mathcal{S}_{\mathscr{T}}^{*}(A, B)$ be functions of the form

$$
f_{k}(z)=z-\sum_{n=1}^{\infty}\left(\left|a_{k, n}\right| z^{n}-\left|b_{k, n}\right| \bar{z}^{n}\right)
$$

and let $0 \leq \gamma \leq 1$. Since

$$
\begin{aligned}
\gamma f_{1}(z) & +(1-\gamma) f_{2}(z) \\
= & z-\sum_{n=2}^{\infty}\left(\gamma\left|a_{1, n}\right|+(1-\gamma)\left|a_{2, n}\right|\right) z^{n} \\
& +\sum_{n=1}^{\infty}\left(\gamma\left|b_{1, n}\right|+(1-\gamma)\left|b_{2, n}\right|\right) \bar{z}^{n}
\end{aligned}
$$

and by Theorem 4 we have

$$
\begin{aligned}
& \sum_{n=1}^{\infty}\left\{\alpha_{n}\left(\gamma\left|a_{1, n}\right|+(1-\gamma)\left|a_{2, n}\right|\right)\right. \\
& \left.\quad+\beta_{n}\left(\gamma\left|b_{1, n}\right|+(1-\gamma)\left|b_{2, n}\right|\right)\right\}=\gamma \sum_{n=1}^{\infty}\left\{\alpha_{n}\left|a_{1, n}\right|\right. \\
& \left.\quad+\beta_{n}\left|b_{1, n}\right|\right\}+(1-\gamma) \sum_{n=1}^{\infty}\left\{\alpha_{n}\left|a_{2, n}\right|+\beta_{n}\left|b_{2, n}\right|\right\} \\
& \quad \leq 2 \gamma(B-A)+2(1-\gamma)(B-A)=2(B-A),
\end{aligned}
$$

the function $\phi=\gamma f_{1}+(1-\gamma) f_{2}$ belongs to the class $\mathcal{S}_{\mathscr{T}}^{*}(A, B)$. Hence, the class is convex. Furthermore, for $f \in$ $\mathcal{S}_{\mathscr{T}}^{*}(A, B),|z| \leq r, 0<r<1$, we have

$$
\begin{aligned}
|f(z)| & \leq \sum_{n=1}^{\infty}\left(\left|a_{n}\right|+\left|b_{n}\right|\right) r^{n} \\
& \leq \sum_{n=1}^{\infty}\left(\frac{\alpha_{n}}{B-A}\left|a_{n}\right|+\frac{\beta_{n}}{B-A}\left|b_{n}\right|\right) \leq 2 .
\end{aligned}
$$

Thus, we conclude that the class $\mathcal{S}_{\mathscr{T}}^{*}(A, B)$ is locally uniformly bounded. By Lemma 15, we only need to show that it is closed; that is, if $f_{k} \in \mathcal{S}_{\mathscr{T}}^{*}(A, B)(k \in \mathbb{N})$ and $f_{k} \rightarrow f$, then $f \in$ $\mathcal{S}_{\mathscr{T}}^{*}(A, B)$. Let $f_{k}$ and $f$ be given by (68) and (5), respectively. Using Theorem 4 we have

$$
\sum_{n=1}^{\infty}\left(\alpha_{n}\left|a_{k, n}\right|+\beta_{n}\left|b_{k, n}\right|\right) \leq 2(B-A), \quad(k \in \mathbb{N}) .
$$

Since $f_{k} \rightarrow f$, we conclude that $a_{k, n} \rightarrow a_{n}$ and $b_{k, n} \rightarrow b_{n}$ as $k \rightarrow \infty \quad(n \in \mathbb{N})$. The sequence of partial sums $\left\{S_{n}\right\}$ associated with the series $\sum_{n=1}^{\infty}\left(\alpha_{n}\left|a_{n}\right|+\beta_{n}\left|b_{n}\right|\right)$ is nondecreasing sequence. Moreover, by (72) it is bounded by $2(B-A)$. Therefore, the sequence $\left\{S_{n}\right\}$ is convergent and

$$
\sum_{n=2}^{\infty}\left(\alpha_{n}\left|a_{n}\right|+\beta_{n}\left|b_{n}\right|\right)=\lim _{n \rightarrow \infty} S_{n} \leq B-A .
$$

This gives condition (24), and, in consequence, $f \in$ $\mathcal{S}_{\mathscr{T}}^{*}(A, B)$, which completes the proof.

Theorem 17. Consider

$$
E \mathcal{S}_{\mathscr{T}}^{*}(A, B)=\left\{h_{n}^{*}: n \in \mathbb{N}\right\} \cup\left\{g_{n}^{*}: n \in \mathbb{N}\right\},
$$


where

$$
\begin{aligned}
& h_{1}^{*}(z)=z, \\
& h_{n}^{*}(z)=z-\frac{B-A}{\alpha_{n}} z^{n}, \\
& g_{n}^{*}(z)=z+\frac{B-A}{\beta_{n}} \bar{z}^{n},
\end{aligned}
$$

$$
(z \in \mathbb{U}) \text {. }
$$

Proof. Suppose that $0<\gamma<1, n \in \mathbb{N}$, and

$$
g_{n}^{*}=\gamma f_{1}+(1-\gamma) f_{2}
$$

where $f_{1}, f_{2} \in \mathcal{S}_{\mathscr{T}}^{*}(A, B)$ are functions of form (8). Then, by (24) we have $\left|b_{1, n}\right|=\left|b_{2, n}\right|=(B-A) / \beta_{n}$, and, in consequence, $a_{1, k}=a_{2, k}=0$ for $k \in\{2,3, \ldots\}$ and $b_{1, k}=b_{2, k}=0$ for $k \in \mathbb{N} \backslash\{n\}$. It follows that $g_{n}^{*}=f_{1}=f_{2}$, and consequently $g_{n}^{*} \in E \mathcal{S}_{\mathscr{T}}^{*}(A, B)$. Similarly, we verify that the functions $h_{n}^{*}$ of form (75) are the extreme points of the class $\mathcal{S}_{\mathscr{T}}^{*}(A, B)$. Now, suppose that $f \in E \mathcal{S}_{\mathscr{T}}^{*}(A, B)$ and $f$ is not of form (75). Then there exists $k \in \mathbb{N}$ such that

$$
\begin{array}{r}
0<\left|a_{k}\right|<\frac{B-A}{\alpha_{k}} \\
\text { or } 0<\left|b_{k}\right|<\frac{B-A}{\beta_{k}} .
\end{array}
$$

If $0<\left|a_{k}\right|<(B-A) / \alpha_{k}$, then, putting

$$
\begin{aligned}
& \gamma=\frac{\left|a_{k}\right| \alpha_{k}}{B-A}, \\
& \varphi=\frac{1}{1-\gamma}\left(f-\gamma h_{k}^{*}\right),
\end{aligned}
$$

we have that $0<\gamma<1, h_{k}^{*}, \varphi \in \mathcal{S}_{\mathscr{T}}^{*}(A, B), h_{k}^{*} \neq \varphi$, and

$$
f=\gamma h_{k}^{*}+(1-\gamma) \varphi \text {. }
$$

Thus, $f \notin E \mathcal{S}_{\mathscr{T}}^{*}(A, B)$. Similarly, if $0<\left|b_{k}\right|<(B-A) / \beta_{k}$, then, putting

$$
\begin{gathered}
\gamma=\frac{\left|b_{k}\right| \beta_{k}}{B-A}, \\
\phi(z)=\frac{1}{1-\gamma}\left(f-\gamma g_{k}^{*}\right),
\end{gathered}
$$

we have that $0<\gamma<1, g_{k}^{*}, \varphi \in \mathcal{S}_{\mathscr{T}}^{*}(A, B), g_{k}^{*} \neq \phi$, and

$$
f=\gamma g_{k}^{*}+(1-\gamma) \phi .
$$

It follows that $f \notin E \mathcal{S}_{\mathscr{T}}^{*}(A, B)$, and the proof is completed.

The following result may be proved in much the same way as Theorems 16 and 17.
Theorem 18. The class $\mathcal{S}_{\mathscr{T}}^{c}(A, B)$ is convex and compact subset of $\mathscr{H}_{0}$. Moreover,

$$
E \mathcal{S}_{\mathscr{T}}^{\mathcal{C}}(A, B)=\left\{h_{n}: n \in \mathbb{N}\right\} \cup\left\{g_{n}: n \in \mathbb{N}\right\}
$$

where

$$
\begin{aligned}
& h_{1}(z)=z, \\
& h_{n}(z)=z-\frac{B-A}{n \alpha_{n}} z^{n}, \\
& g_{n}(z)=z-\frac{B-A}{n \beta_{n}} \bar{z}^{n},
\end{aligned}
$$

$(z \in \mathbb{U})$.

\section{Applications}

It is clear that for the locally uniformly bounded class

$$
\mathscr{F}=\left\{f_{n} \in \mathscr{H}: n \in \mathbb{N}\right\}
$$

we have

$$
\overline{\mathrm{co}} \mathscr{F}=\left\{\sum_{n=1}^{\infty} \gamma_{n} f_{n}: \sum_{n=1}^{\infty} \gamma_{n}=1, \gamma_{n} \geq 0 \quad(n \in \mathbb{N})\right\} .
$$

Thus, by Theorems 16, 17, and 18 we have the following two corollaries.

\section{Corollary 19. Consider}

$$
\begin{aligned}
& \mathcal{S}_{\mathscr{T}}^{*}(A, B)=\left\{\sum_{n=1}^{\infty}\left(\gamma_{n} h_{n}^{*}+\delta_{n} g_{n}^{*}\right): \sum_{n=1}^{\infty}\left(\gamma_{n}+\delta_{n}\right)\right. \\
& \left.\quad=1, \gamma_{n}, \delta_{n} \geq 0\right\},
\end{aligned}
$$

where $h_{n}^{*}, g_{n}^{*}$ are defined by (75).

Corollary 20. Consider

$$
\begin{aligned}
& \mathcal{S}_{\mathscr{T}}^{c}(A, B)=\left\{\sum_{n=1}^{\infty}\left(\gamma_{n} h_{n}+\delta_{n} g_{n}\right): \sum_{n=1}^{\infty}\left(\gamma_{n}+\delta_{n}\right)\right. \\
& \left.\quad=1, \gamma_{n}, \delta_{n} \geq 0\right\},
\end{aligned}
$$

where $h_{n}, g_{n}$ are defined by (83). 
For each fixed value of $n \in \mathbb{N}, z \in \mathbb{U}$, the following realvalued functionals are continuous and convex on $\mathscr{H}$ :

$$
\begin{aligned}
& \mathscr{J}(f)=\left|a_{n}\right|, \\
& \mathscr{J}(f)=\left|b_{n}\right|, \\
& \mathscr{J}(f)=|f(z)|, \\
& \mathscr{J}(f)=\left|D_{\mathscr{H}} f(z)\right| \\
& \mathscr{J}(f)=\left(\frac{1}{2 \pi} \int_{0}^{2 \pi}\left|f\left(r e^{i \theta}\right)\right|^{\gamma} d \theta\right)^{1 / \gamma}, \\
& \quad(f \in \mathscr{H}, \quad \gamma \geq 1,0<r<1) .
\end{aligned}
$$

Therefore, by Lemma 14 and Theorems 17 and 18 we have the corollaries listed below.

Corollary 21. Let $f \in \mathcal{S}_{\mathscr{T}}^{*}(A, B)$ be a function of form (35). Then

$$
\begin{aligned}
& \left|a_{n}\right| \leq \frac{B-A}{\alpha_{n}}, \\
& \left|b_{n}\right| \leq \frac{B-A}{\beta_{n}},
\end{aligned}
$$

$(n \in \mathbb{N})$

where $\alpha_{n}, \beta_{n}$ are defined by (25). The result is sharp. The functions $h_{n}^{*}, g_{n}^{*}$ of form (75) are the extremal functions.

Corollary 22. Let $f \in \mathcal{S}_{\mathscr{T}}^{c}(A, B)$ be a function of form (35). Then

$$
\begin{aligned}
& \left|a_{n}\right| \leq \frac{B-A}{n \alpha_{n}}, \\
& \left|b_{n}\right| \leq \frac{B-A}{n \beta_{n}}
\end{aligned}
$$

$(n \in \mathbb{N})$

where $\alpha_{n}, \beta_{n}$ are defined by (25). The result is sharp. The functions $h_{n}, g_{n}$ of form (83) are the extremal functions.

Corollary 23. Let $f \in \mathcal{S}_{\mathscr{T}}^{*}(A, B),|z|=r<1$. Then

$$
\begin{aligned}
& r-\frac{B-A}{1+2 B-A} r^{2} \leq|f(z)| \leq r+\frac{B-A}{1+2 B-A} r^{2}, \\
& r-\frac{2(B-A)}{1+2 B-A} r^{2} \leq\left|D_{\mathscr{H}} f(z)\right| \leq r+\frac{2(B-A)}{1+2 B-A} r^{2} .
\end{aligned}
$$

The result is sharp. The function $h_{2}^{*}$ of form (75) is the extremal function.
Corollary 24. Let $f \in \mathcal{S}_{\mathscr{T}}^{c}(A, B)$ and $|z|=r<1$. Then

$$
\begin{aligned}
r-\frac{B-A}{2(1+2 B-A)} r^{2} & \leq|f(z)| \\
& \leq r+\frac{B-A}{2(1+2 B-A)} r^{2}, \\
r-\frac{B-A}{1+2 B-A} r^{2} & \leq\left|D_{\mathscr{H}} f(z)\right| \\
& \leq r+\frac{B-A}{1+2 B-A} r^{2} .
\end{aligned}
$$

The result is sharp. The function $h_{2}$ of form (83) is the extremal function.

Due to Littlewood [12] we obtain the integral means inequalities for functions from the classes $\mathcal{S}_{\mathscr{T}}^{*}(A, B)$, $\mathcal{S}_{\mathscr{T}}^{c}(A, B)$.

Lemma 25 (see [12]). Let $f, g \in \mathscr{A}$. If $f \prec g$, then

$$
\begin{aligned}
\int_{0}^{2 \pi}\left|f\left(r e^{i \theta}\right)\right|^{\gamma} d \theta \leq \int_{0}^{2 \pi}\left|g\left(r e^{i \theta}\right)\right|^{\gamma} d \theta, & \\
& (0<r<1, \gamma>0) .
\end{aligned}
$$

Let $h_{n}^{*}$ and $g_{n}^{*}$ be defined by (75) and let $\tilde{g}_{n}(z)=z+((B-$ $\left.A) / \beta_{n}\right) z^{n}(n \in \mathbb{N})$. Since $h_{n}^{*}(z) / z \prec h_{2}^{*}(z) / z$ and $\tilde{g}(z) / z \prec$ $h_{2}^{*}(z) / z$, by Lemma 25 we have

$$
\begin{aligned}
\int_{0}^{2 \pi}\left|\frac{h_{n}^{*}(z)}{z}\right|^{\gamma} d \theta & \leq \int_{0}^{2 \pi}\left|\frac{h_{2}^{*}(z)}{z}\right|^{\gamma} d \theta, \quad\left(z=r e^{i \theta}\right), \\
\int_{0}^{2 \pi}\left|\frac{g_{n}^{*}(z)}{z}\right|^{\gamma} d \theta & =\int_{0}^{2 \pi}\left|\frac{\widetilde{g}_{n}(z)}{z}\right|^{\gamma} d \theta \\
& \leq \int_{0}^{2 \pi}\left|\frac{h_{2}^{*}(z)}{z}\right|^{\gamma} d \theta, \quad\left(z=r e^{i \theta}\right) .
\end{aligned}
$$

Thus, we have the following lemma.

Lemma 26. Let $0<r<1, \gamma>0, n \in \mathbb{N}$. Then

$$
\begin{aligned}
& \frac{1}{2 \pi} \int_{0}^{2 \pi}\left|h_{n}^{*}\left(r e^{i \theta}\right)\right|^{\gamma} d \theta \leq \frac{1}{2 \pi} \int_{0}^{2 \pi}\left|h_{2}^{*}\left(r e^{i \theta}\right)\right|^{\gamma} d \theta \\
& \frac{1}{2 \pi} \int_{0}^{2 \pi}\left|g_{n}^{*}\left(r e^{i \theta}\right)\right|^{\gamma} d \theta \leq \frac{1}{2 \pi} \int_{0}^{2 \pi}\left|h_{2}^{*}\left(r e^{i \theta}\right)\right|^{\gamma} d \theta
\end{aligned}
$$

where $h_{n}^{*}$ and $g_{n}^{*}$ are defined by (75).

By Lemmas 14 and 26 and Theorem 17 we have the following corollary. 
Corollary 27. Let $0<r<1, \gamma \geq 1$. If $f \in \mathcal{S}_{\mathscr{T}}^{*}(A, B)$, then

$$
\begin{aligned}
& \frac{1}{2 \pi} \int_{0}^{2 \pi}\left|f\left(r e^{i \theta}\right)\right|^{\gamma} d \theta \leq \frac{1}{2 \pi} \int_{0}^{2 \pi}\left|h_{2}^{*}\left(r e^{i \theta}\right)\right|^{\gamma} d \theta, \\
& \frac{1}{2 \pi} \int_{0}^{2 \pi}\left|D_{\mathscr{C}} f\left(r e^{i \theta}\right)\right|^{\gamma} d \theta \\
& \quad \leq \frac{1}{2 \pi} \int_{0}^{2 \pi}\left|D_{\mathscr{H}} h_{2}^{*}\left(r e^{i \theta}\right)\right|^{\gamma} d \theta,
\end{aligned}
$$

where $h_{2}^{*}$ is the function defined by (75).

In a similar manner, we can prove Corollary 23 below.

Corollary 28. Let $0<r<1, \gamma \geq 1$. If $f \in \mathcal{S}_{\mathscr{T}}^{c}(A, B)$, then

$$
\begin{aligned}
& \frac{1}{2 \pi} \int_{0}^{2 \pi}\left|f\left(r e^{i \theta}\right)\right|^{\gamma} d \theta \leq \frac{1}{2 \pi} \int_{0}^{2 \pi}\left|h_{2}\left(r e^{i \theta}\right)\right|^{\gamma} d \theta, \\
& \frac{1}{2 \pi} \int_{0}^{2 \pi}\left|D_{\mathscr{H}} f\left(r e^{i \theta}\right)\right|^{\gamma} d \theta \\
& \quad \leq \frac{1}{2 \pi} \int_{0}^{2 \pi}\left|D_{\mathscr{H}} h_{2}\left(r e^{i \theta}\right)\right|^{\gamma} d \theta,
\end{aligned}
$$

where $h_{2}$ is the function defined by (83).

The following covering results follow from Corollaries 23 and 24 .

Corollary 29. If $f \in \mathcal{S}_{\mathscr{T}}^{*}(A, B)$, then

$$
\left\{w:|w|<\frac{1+B}{1+2 B-A}\right\} \subset f(\mathbb{U}) .
$$

Corollary 30. If $f \in \mathcal{S}_{\mathscr{T}}^{c}(A, B)$, then

$$
\left\{w:|w|<\frac{2+3 B-A}{2(1+2 B-A)}\right\} \subset f(\mathbb{U}) \text {. }
$$

Remark 31. By choosing the parameters in the defined classes of functions we can obtain new and also well-known results (see, e.g., $[5-7,10])$.

\section{Conclusion}

In the paper new classes of univalent harmonic functions are introduced. Necessary and sufficient conditions for defined classes of functions are obtained. Some topological properties, radii of convexity and starlikeness, and extreme points of the classes are also considered. By using extreme points theory we obtained coefficients estimates, distortion theorems, and integral mean inequalities for these classes of functions.

\section{Conflict of Interests}

The author declares that there is no conflict of interests regarding the publication of this paper.

\section{Acknowledgment}

The work is supported by the Centre for Innovation and Transfer of Natural Sciences and Engineering Knowledge, University of Rzeszów.

\section{References}

[1] H. Lewy, "On the non-vanishing of the Jacobian in certain one-to-one mappings," Bulletin of the American Mathematical Society, vol. 42, no. 10, pp. 689-692, 1936.

[2] J. Clunie and T. Sheil-Small, "Harmonic univalent functions," Annales Academiae Scientiarum Fennicae. Series A I. Mathematica, vol. 9, pp. 3-25, 1984.

[3] T. Sheil-Small, "Constants for planar harmonic mappings," Journal London Mathematical Society, vol. 2, no. 42, pp. $237-$ 248, 1990.

[4] W. Janowski, "Some extremal problems for certain families of analytic functions I," Annales Polonici Mathematici, vol. 28, pp. 297-326, 1973.

[5] J. M. Jahangiri, "Coefficient bounds and univalence criteria for harmonic functions with negative coefficients," Annales Universitatis Mariae Curie-Skłodowska A, vol. 52, no. 2, pp. 5766, 1998.

[6] J. M. Jahangiri, "Harmonic functions starlike in the unit disk," Journal of Mathematical Analysis and Applications, vol. 235, no. 2, pp. 470-477, 1999.

[7] H. Silverman, "Harmonic univalent functions with negative coefficients," Journal of Mathematical Analysis and Applications, vol. 220, no. 1, pp. 283-289, 1998.

[8] M. Krein and D. Milman, "On the extreme points of regularly convex sets," Studia Mathematica, vol. 9, no. 1, pp. 133-138, 1940.

[9] D. J. Hallenbeck and T. H. MacGregor, Linear Problems and Convexity Techniques in Geometric Function Theory, Pitman Advanced Publishing Program, Pitman, Boston, Mass, USA, 1984.

[10] J. Dziok, “On janowski harmonic functions,” Journal of Applied Analysis, vol. 21, no. 2, 2015.

[11] P. Montel, "Sur les families de functions analytiques qui admettent des valeurs exceptionelles dans un domaine," Annales Scientifiques de lÉcole Normale Supérieure, vol. 23, pp. 487-535, 1912.

[12] J. E. Littlewood, "On inequalities in theory of functions," Proceedings London Mathematical Society, vol. s2-23, no. 1, pp. 481-519, 1925. 


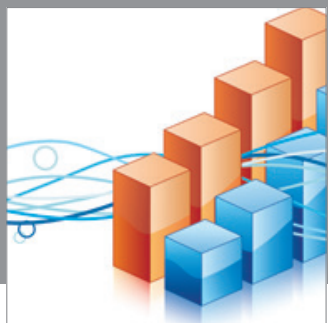

Advances in

Operations Research

mansans

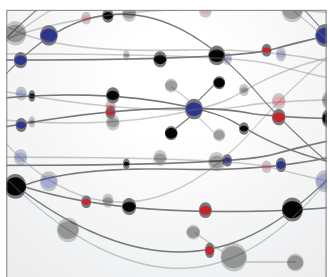

The Scientific World Journal
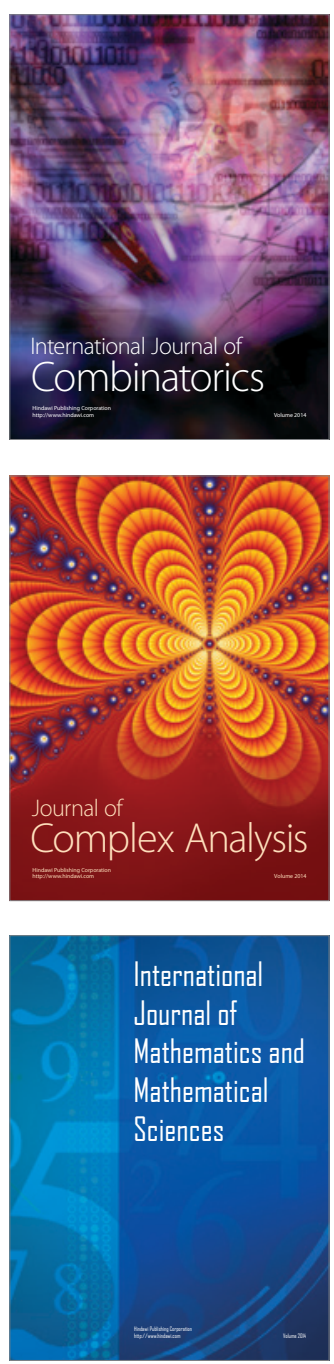
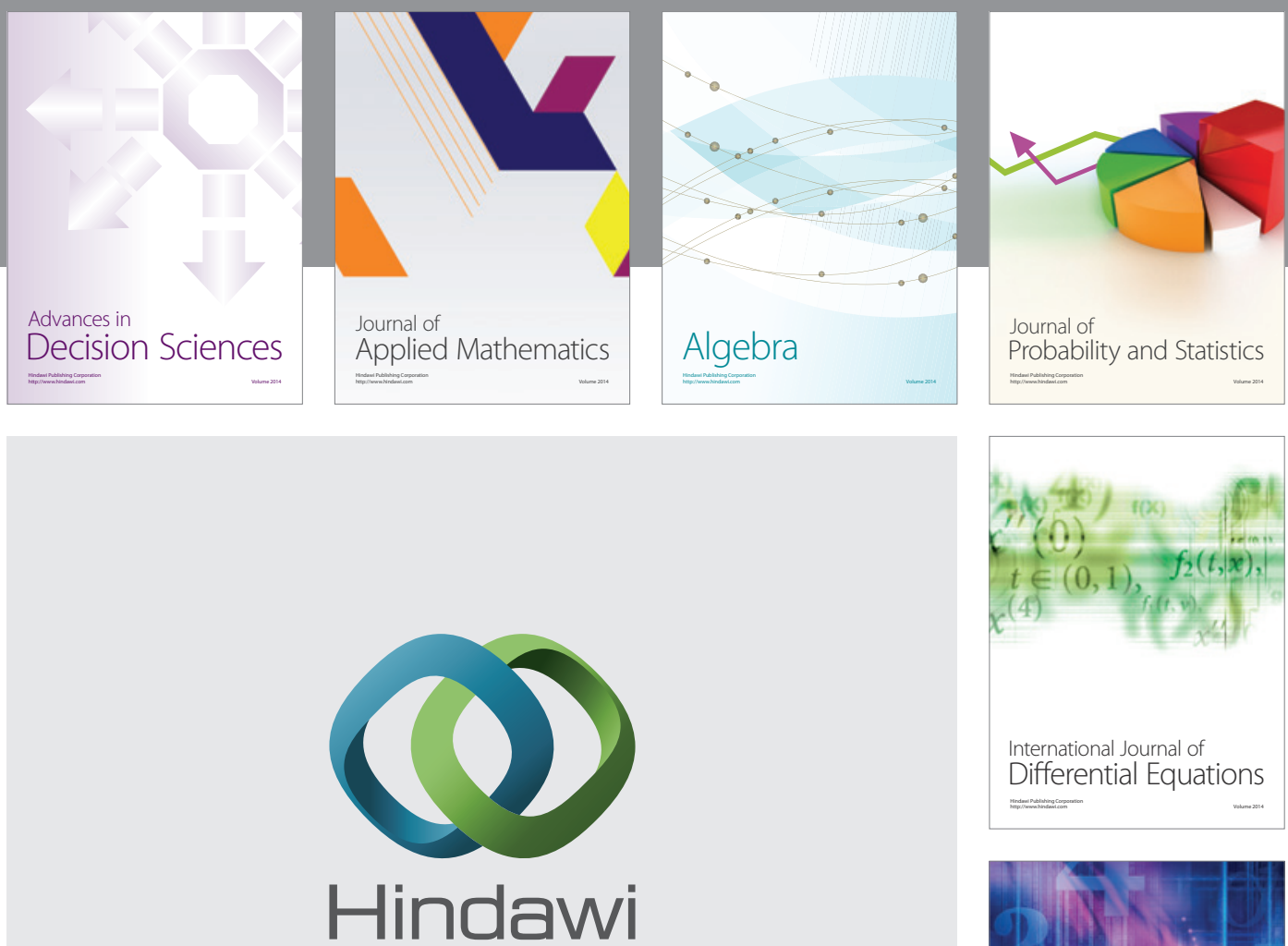

Submit your manuscripts at http://www.hindawi.com
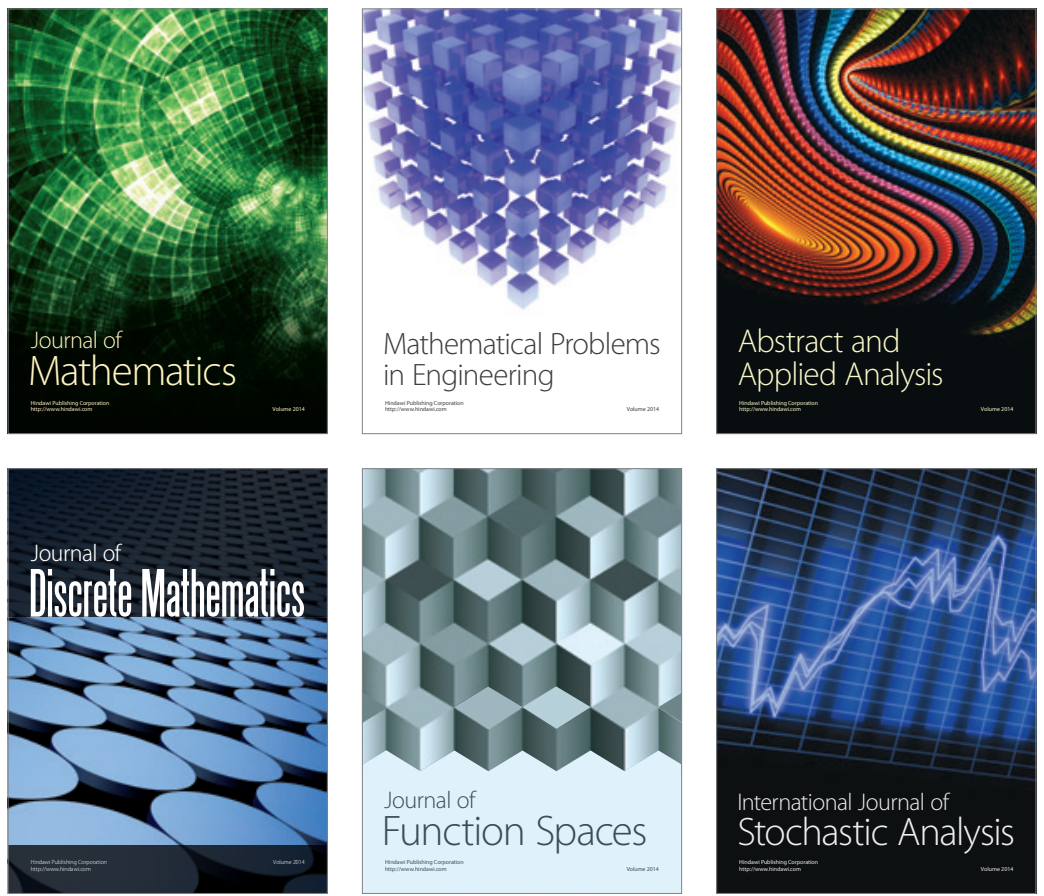

Journal of

Function Spaces

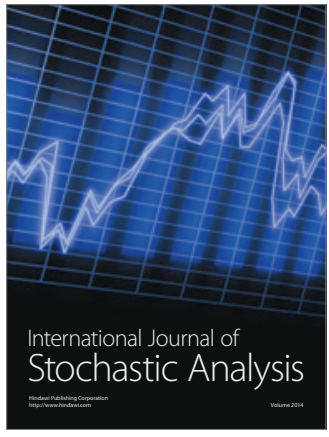

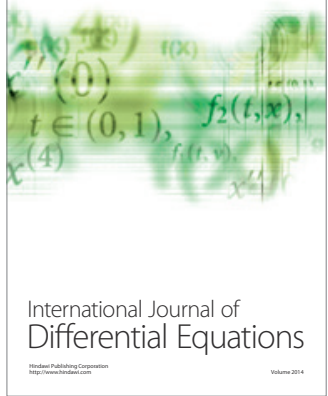
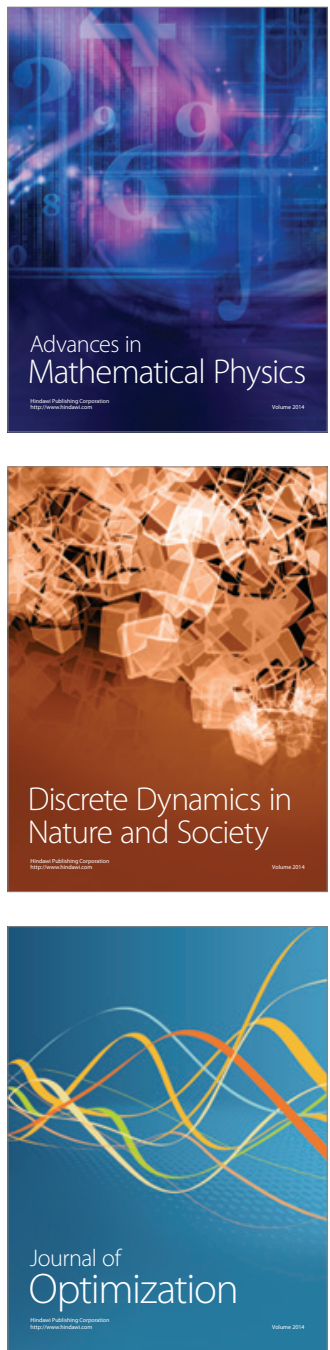